\title{
Hierarchical Discrete Medial Axis for Sphere-Tree Construction
}

\author{
Alain Broutta, David Coeurjolly, Isabelle Sivignon \\ Université de Lyon CNRS \\ Laboratoire LIRIS - UMR 5205 F-69622 Villeurbanne Cedex, France \\ E-mail : alain.broutta@liris.cnrs.fr
}

\begin{abstract}
In discrete geometry, the Distance Transformation and the Medial Axis Extraction are classical tools for shape analysis. In this paper, we present a Hierarchical Discrete Medial Axis, based on a pyramidal representation of the object, in order to efficiently create a sphere-tree, which has many applications in collision detection or image synthesis.
\end{abstract}

\section{Introduction}

In interactive environments, hierarchical representations are classical tools with many applications, e.g. in image synthesis, multi-resolution representations and interference detection, as they allow a fast targeting of interaction. The Bounding Volume Hierarchies $(\mathrm{BVH})$ consist in coverage with an increasing number of simple volumes (spheres [17,14,9], axis-aligned bounding boxes [18], oriented bounding boxes $[11], \ldots$ ) on different levels, respecting the fact that each part of the object covered by a children node must be covered by the parent node. Hence the collision detection between two hierarchical models is computed by a recursive process of intersection tests on pair of primitive volumes. The choice of the primitive volume is important for the BVH properties [11]. Sphere-trees are very interesting for interference detection. Nevertheless, as they are bad estimators of the object geometry, the hierarchy need an effective construction of the sphere-tree, reducing the error between the object and the associated set of spheres.

In computational geometry, first algorithms for sphere-tree construction were based on Octree data structures $[14,9,12]$, which consist in recursive spatial subdivisions of the object. Then, efficient algorithms are based on the object Medial Axis (MA) [4], which corresponds to a skeleton representation of the object. MA extraction is based a Voronoi Diagram [13, 6, 7], and the sphere-tree is produced by complex optimization heuristics to reduce the sphere number, with a control on the error (approximated Haussdorf distance) and its distribution around the object.

In discrete geometry, the exact Discrete Medial Axis (DMA) can be efficiently computed from using Distance Transformation (DT) [8]. DMA is a convenient tool to represent objects in digital space, since it is reversible: from the DMA points, we can exactly reconstruct the original shape. The study of multiresolution representation of digital objects has been carried out, using an homotopic 
thinning [16]. Our goal is to develop a hierarchical structure which is flexible with respect to the reversibility of the construction. Furthermore, a discrete approach benefits from the fact that the error between the object and the hierarchy (at each level) can be exactly computed with a Hamming distance.

In this paper, we present an original method for sphere-tree construction (sketched in Figure 1), where the sets of spheres at each level are obtained by a DMA extraction at different object resolution levels, in a regular pyramidal approach. Preliminaries are presented in Section 2. As the set of spheres is reversible we efficiently control the error propagation and its distribution at each level. The sphere-tree construction is achieved by linking the spheres on consecutive levels. This method is first defined for a reversible model for volume synthesis (Sections 3.1-3.2), however it can be easily adapted for interactive environments (Section 3.3). Experiments in Section 4 also show that this last modification reduces the error. Finally we obtain a $d$-dimensional generic sphere-tree computation in linear time for any discrete distance and pyramidal model.

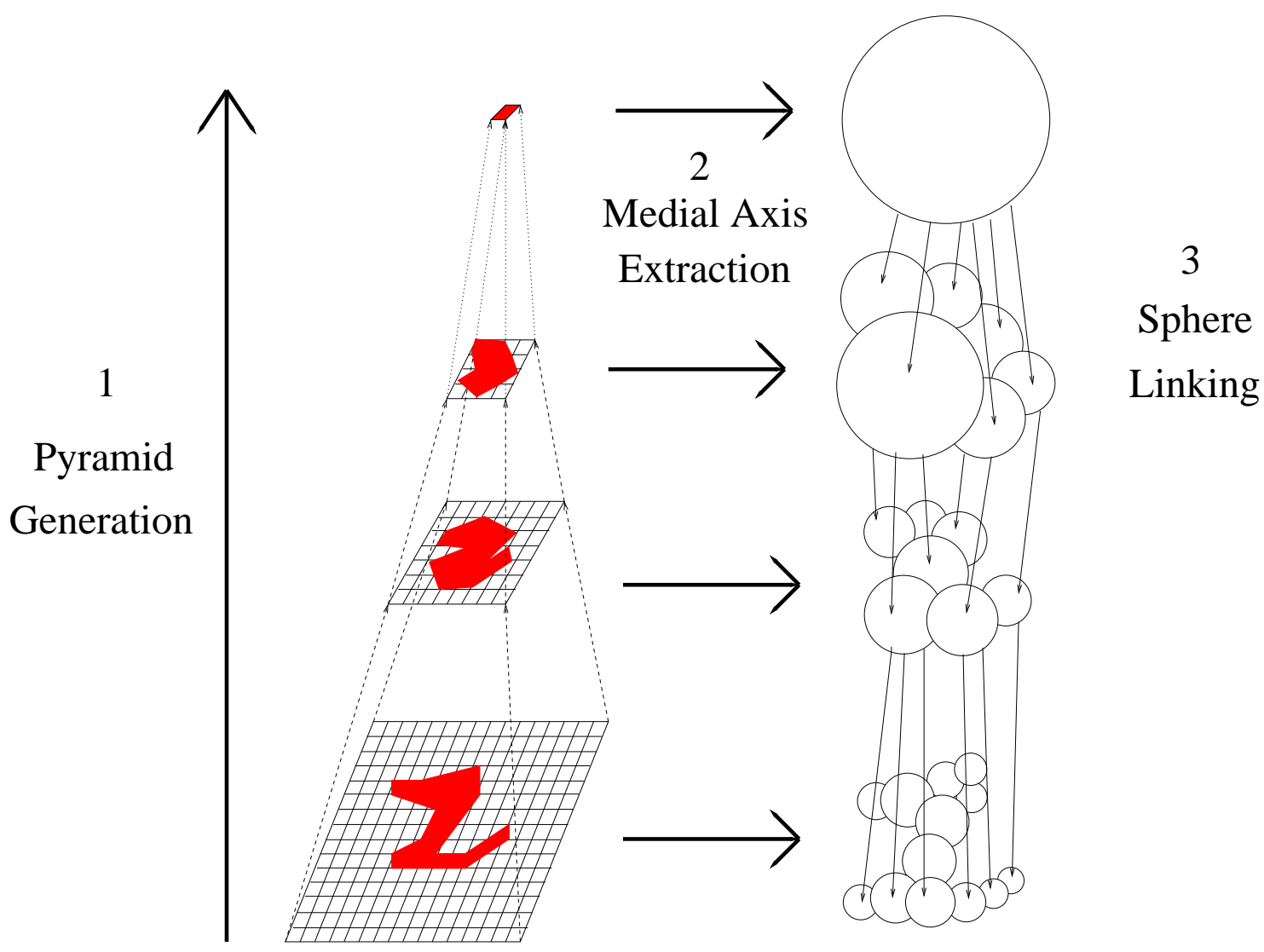

Fig. 1. The main stages of the sphere-tree construction. 


\section{Preliminaries}

Contrary to previous algorithms described in computational geometry, our method aims at controlling the error increasing and its distribution at each level, by a pyramid construction on the original object. Then the DMA extraction computed a reversible set of spheres.

\subsection{Pyramidal Model}

In image analysis, pyramidal structure is a convenient tool in image analysis and segmentation. We can simply define a pyramid $\mathcal{P}$ of depth $N+1$ by a set of $2 \mathrm{D}$ images $\left\{\mathcal{F}_{0}, \ldots, \mathcal{F}_{N}\right\}$, where $\mathcal{F}_{N}$ represent the original image with at finer resolution. The regular pyramid construction is a bottom-up process: the upper levels are representations of $\mathcal{F}_{N}$ with a lower resolution in a quad-tree approach; the pixel size at each level is 4 time bigger than at next level. The pixel color is based on the 4 pixels and computed by a transform function (cf Figure 2).

We can generalize this process to any $d$ dimensions with an integer factor $f$ for the voxel size expansion. In other words, one voxel $v^{\prime}$ from level $L-1$ contains $f^{d}$ voxels $\left\{v_{1}, \ldots, v_{f^{d}}\right\}$ at level $L$. So, the transfer function, denoted $\mathcal{M}$ (standing for Model), is a relation between these voxels:

Definition 1. $\mathcal{M}$ is a model between $\mathcal{F}_{L}$ and $\mathcal{F}_{L-1}\left(\mathcal{F}_{L-1}=\mathcal{M}\left(\mathcal{F}_{L}\right)\right)$ if $\forall v^{\prime}, \exists\left\{v_{1}, \ldots, v_{f^{d}}\right\} \backslash \mathcal{F}_{L-1}\left(v^{\prime}\right)=\mathcal{M}\left(\mathcal{F}_{L}\left(v_{1}\right), \ldots \mathcal{F}_{L}\left(v_{f^{d}}\right)\right)$

In our future bounding volume hierarchy approach, we need that there is no loss of information, in other words, the original object must be completely covered at each level of the hierarchy. So we choose including models, where each representation at any level $L$ is included in the above representation at level $L-1$. For example, we propose a model which can be seen as the logic operand "OR": Each voxel at level $L-1$ belongs to $\mathcal{F}_{L-1}$ when at least one of the $f^{d}$ voxels that it contains (at level $L$ ) belongs to $\mathcal{F}_{L}$. Figure 2 shows an application of this model on 2D images.
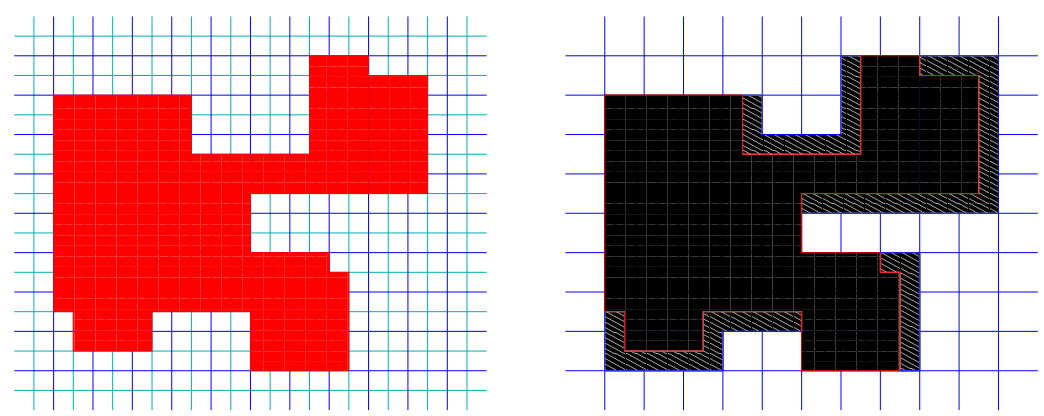

Fig. 2. Consecutive levels on a regular pyramid with the OR-Model. 


\subsection{Discrete Medial Axis and Discrete Power Diagram}

Discrete Medial Axis. The Medial Axis is a shape descriptor first presented by Blum in 1967 [4] in order to simulate the wavefront propagation initiated on its boundary (prairie fire model). The medial axis is defined by (1) the locus of of points equidistant from two side of the object, or (2) the locus of centers of maximals balls included in the object (a ball is maximal if it is not included in any another ball included in the object) [15].

In binary images, the Discrete Medial Axis (DMA) can be efficiently extracted from a Distance Transformation (DT). It consists of labeling each voxel of an object with distance of the closest voxel of the complement (background). In other words, the DT value on a voxel $v$ corresponds to the radius of the largest discrete ball centered in $s$ included in the object. So, we can efficiently detect the maximal balls.

Discrete Power Diagram. In computational geometry, the Power diagram (also known as the Laguerre diagram) is a generalization of the Voronoi Diagram [3]. This tool is widely used for ball interaction computation and surface reconstruction $[5,2,1]$. We consider a set of sites $\mathcal{S}=\left\{s_{i}\right\}$, such that each point $s_{i}$ is associated with a radius $r_{i}$. The power $\sigma_{i}(p)$ of a point $p$ according to the site $s_{i}$ is given by:

$$
\sigma_{i}(p)=d\left(p, s_{i}\right)-r_{i}
$$

If $\sigma_{i}(p)<0, p$ belongs to the ball of center $s_{i}$ and radius $r_{i}$. The Power diagram is based on the metric induced by $\sigma$ and it is a decomposition of the object into cells $\mathcal{C}=\left\{c_{i}\right\}$ associated with each site $s_{i}$ such that:

$$
c_{i}=\left\{p \in \mathbb{R}^{d}: \sigma_{i}(p)<\sigma_{j}(p), i \neq j\right\}
$$

In discrete geometry, the power labeling is defined as the power diagram labeling of grid points [8]. More precisely, we assign to each grid point of the plane the index of the cell containing it in the power diagram, so the discrete power diagram is a labelling for each voxel by the sphere which best covers it.

\section{Sphere-tree construction}

The previous section has presented convenient tools for the construction of a sphere-tree whose the set of nodes at each level results from the DMA extraction at each pyramid level. In complete our sphere-tree process, we still have to link the spheres at consecutive levels.

\subsection{Power diagram and Sphere-DAG}

On this first approach, we propose an exhaustive linking of spheres on two consecutive levels, in order to compute a sphere-tree by extracting its minimal spanning tree. The most simple relationship between a sphere $s$ at level $N$ and a sphere $t$ 
at level $N-1$ can be defined by the covering of a part of $s$ by $t$. We note $t \rightarrow s$ an edge between $t$ and $s$ :

$$
t \rightarrow s \Leftrightarrow t \cap s \neq \emptyset
$$

As these edges are always oriented from a sphere $t$ to a sphere $s$ at next level, the graph defined by the equation is a Direct Acyclic Graph (DAG), where nodes are DMA spheres at different levels. The construction of this Sphere-DAG needs to test the intersection between two spheres at one level and at the previous level, so the time for the computation is in $O\left(n^{2}\right)$ for $n$ spheres at the lower level. However, in a correct hierarchy, each part of the object does not need to be covered by a large set of spheres, so this Sphere-DAG is too exhaustive for an efficient sphere-tree simplification. In order to reduce the number of spheres of the complete Sphere-DAG, we use the discrete power diagram, defined in 2.2, as it is a voxel labeling by the sphere which best covers it. Let $v$ be a voxel of $\mathcal{F}_{N}$ at level $N$. As we built the upper representation $\mathcal{F}_{N-1}$ at level $N-1$ with a model $\mathcal{M}, v$ is included in a voxel $v^{\prime}$. We note $v^{\prime}=\mathcal{S}(v)$. Including models, like the "OR" model defined in 2.1, ensure that $\mathcal{F}_{N-1}\left(v^{\prime}\right)=1$. So we can compare the power diagrams $\mathcal{C}_{N}$ (from $\mathcal{F}_{N}$ ) and $\mathcal{C}_{N-1}\left(\right.$ from $\mathcal{F}_{N-1}$ ). If $v$ belongs to the cell $\mathcal{C}_{N}(s)$ and $v^{\prime}$ to $\mathcal{C}_{N-1}(t)$, the sphere $s_{i}$ (at level $N$ ) covers a part of $\mathcal{F}_{N}$ which include at least the voxel $v$, and the representation of this part in $\mathcal{F}_{N-1}$ is covered by the sphere $t_{j}$. In other words, $t_{j}$ covers a part of $s_{i}$, and we represent it by linking $t_{j}$ and $s_{i}$.

Definition 2. $t$ is a parent sphere for $s(t \rightarrow s)$ in the Sphere-DAG if $\exists v \in \mathcal{F}_{N} \backslash\left(v \in \mathcal{C}_{N}(s) \wedge \mathcal{S}(v) \in \mathcal{C}_{N-1}(t)\right)$

By an overlapping of $\mathcal{C}_{N}$ and $\mathcal{C}_{N-1}$ we can detect all relations by only one scan on each voxels at level $N$. For example, in Figure 3, the cell $s_{1}$ in $\mathcal{C}_{N}$ is covered by both $t_{1}$ and $t_{2}$ in $\mathcal{C}_{N-1}$, so we set $t_{1} \rightarrow s_{1}, t_{2} \rightarrow s_{1}$. The algorithm 1 is generic for objects in dimension $d$ and for any including pyramidal model.
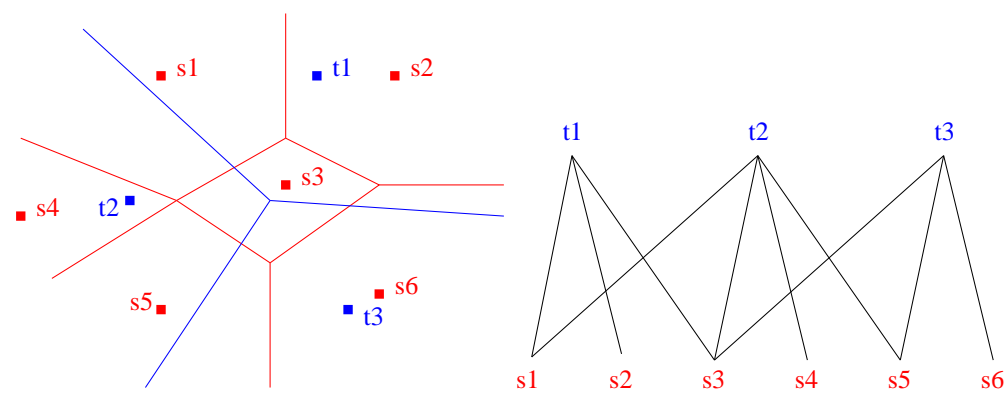

Fig. 3. Overlapping of power diagrams $\mathcal{C}_{N}$ (in red) et $\mathcal{C}_{N-1}$ (in blue) and levels in the associated part of the sphere DAG. 


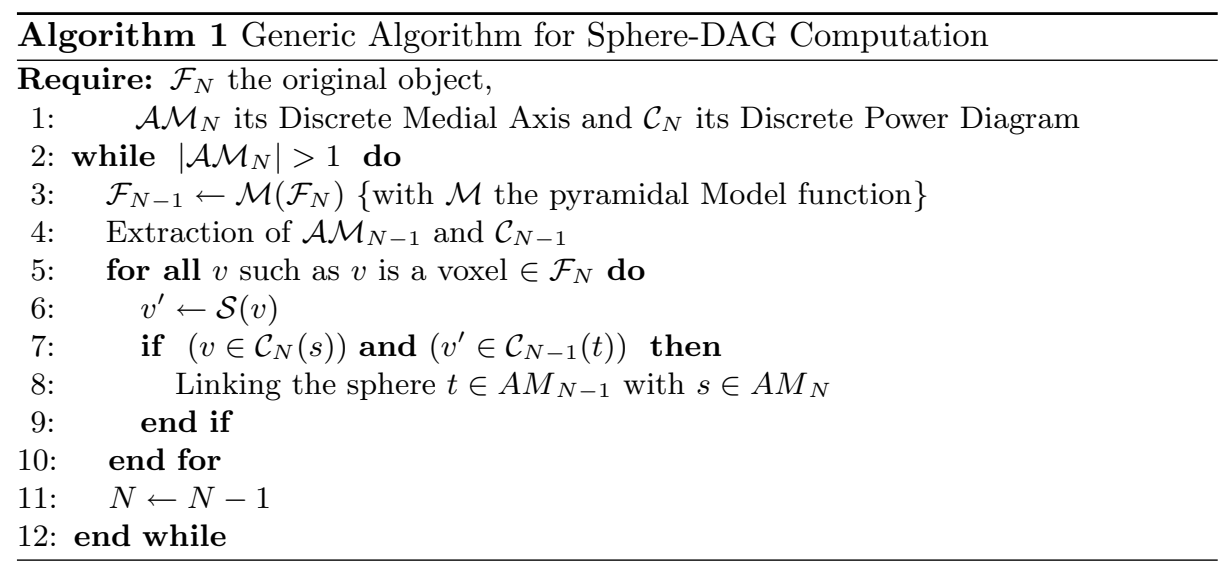

Theorem 1. The generic Sphere-DAG construction process is linear in the number of voxels in $\mathcal{F}_{N}\left(O\left(c^{d}\right)\right)$.

Proof. We consider that the original object $\mathcal{F}_{N}$ is composed by $m$ voxels (or $c^{d}$ voxels if the object is bounded by a cube with side $c$ in $d$ dimensions). The Medial Axis extraction and power diagram computation are in $O\left(c^{d}\right)$, as the construction of $\mathcal{F}_{N-1}$ and the linking between the two sets of spheres, which need a pass on each voxel of $\mathcal{F}_{N}$. So the computation of one iteration of the while loop $\left(\mathcal{F}_{N-1}\right.$ construction and sphere linking) is in $O\left(c^{d}\right)$. Next, we work with the object $\mathcal{F}_{N-1}$, whose size is $f^{d}$ times smaller, and the computation of $\mathcal{F}_{N-2}$ is in $O\left(\frac{c^{d}}{f^{d}}\right)$, and so on. Thus the overall computation time of the whole hierarchy is given by the geometric series $c^{d}+\frac{c^{d}}{f^{d}}+\frac{c^{d}}{\left(f^{d}\right)^{2}}+\ldots=c^{d} \sum_{i=0}^{N} \frac{1}{\left(f^{d}\right)^{i}}$ bounded by $\frac{c^{d}}{1-\frac{1}{f^{d}}}=c^{d} \frac{f^{d}}{f^{d}-1}$. Hence the generic Sphere-DAG computation process is linear in the number of voxels in $\mathcal{F}_{N}$.

\subsection{Reversible Sphere-Tree}

To compute a sphere-tree from the sphere-DAG, we extract a minimal spanning tree, keeping the parent sphere for each node which best covers it (cf Figure 4).

Usually, in a bounding volume hierarchy each node has to cover the union of parts of the object covered by its children nodes, and not the whole volume of each child. Here, our spheres result from DMA extraction ensuring the fact that they cover a part of the object without error. So, each sphere has to be completely covered by (at least) one parent sphere at upper level. We can easily determine if a sphere $s_{1}\left(c_{s_{1}}, r_{s_{1}}\right)$ is covered by another sphere $s_{2}\left(c_{s_{2}}, r_{s_{2}}\right)$, comparing the radius $r_{s_{2}}$ with the distance between centers $d\left(c_{s_{1}}, c_{s_{2}}\right)$ added to the radius $r_{s_{1}}$.

$$
s_{1} \subseteq s_{2} \Leftrightarrow d\left(c_{s_{1}}, c_{s_{2}}\right)+r_{s_{1}}-r_{s_{2}} \leq 0
$$



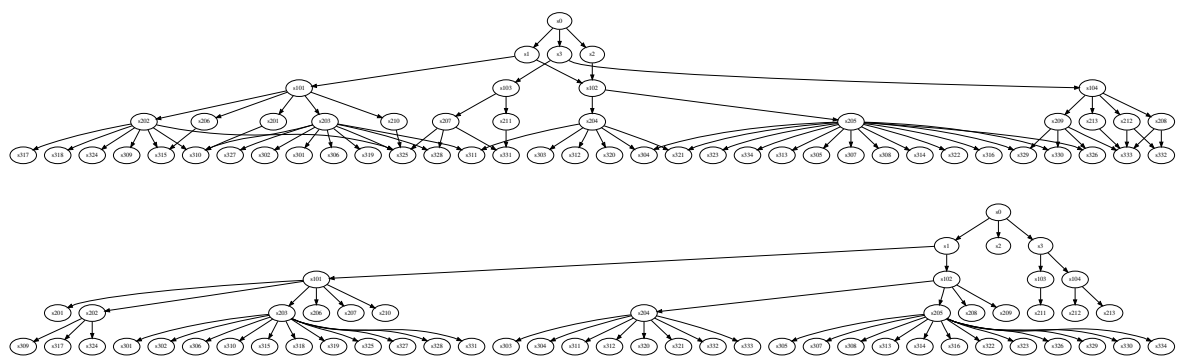

Fig. 4. A sphere DAG and its sphere-tree reduction (here for an ellipsoid).

However it is not sufficient if we want to know if the sphere is covered by more than one sphere. We reformulate the previous covering relation by adapt the $\sigma$ function, defined in the power diagram description (in Section 2.2):

Definition 3. let $s\left(c_{s}, r_{s}\right)$ a sphere at level $N$ and $t\left(c_{t}, r_{t}\right)$ at level $N-1$, the covering power $\sigma_{t}^{\prime}(s)$ is given by

$$
\sigma_{t}^{\prime}(s)=d\left(\mathcal{S}\left(c_{s}\right), c_{t}\right)-r_{t}+r_{s}
$$

If $\sigma_{t}^{\prime}(s) \leq 0$ then $s$ is entirely covered by $t$. More precisely, the covering of $s$ by $t$ is important when $\sigma_{t}^{\prime}(s)$ is small. So, for a children sphere $s$ with $p$ parents $t_{1}, \ldots, t_{p}$, we choose the parent $t_{j}$ where $\sigma_{t_{j}}^{\prime}(s)$ is minimal, in order to get the best coverage of $s$. In fact, we can immediately determine the best parent: for a sphere $s_{i}$ with radius $r_{s_{i}}$ we can only search the minimum of the quantity $d\left(\mathcal{S}\left(c_{s_{i}}\right), c_{t_{j}}\right)-r_{t_{j}}$ for each parent $t_{j}$. With the computation of the power diagram $\mathcal{C}_{N-1}$, it corresponds to the function $\sigma_{t_{j}}\left(\mathcal{S}\left(c_{s_{i}}\right)\right)$ of the point $\mathcal{S}\left(c_{s_{i}}\right)$ for the site $t_{j}$. The minimum of $\sigma$ is reached at $t_{j}$ if the point $\mathcal{S}\left(c_{s_{i}}\right)$ is included in the cell associated to $t_{j}$. Hence, for each sphere $s_{i}$ at level $N$, we just need to detect the position of $\mathcal{S}\left(c_{s_{i}}\right)$, which represents the center $c_{s_{i}}$ at level $N-1$. If $\mathcal{S}\left(c_{s_{i}}\right)$ belongs to the cell associated to the sphere $t_{j}$ then $t_{j}$ is the parent sphere of $s_{i}$. Figure 5 shows this computation in the overlapped power diagrams of Figure 3.

\section{3 $\quad$ Extended Sphere-Tree}

The generated sphere-tree still not guarantee the covering condition for one sphere on its children. Indeed, sphere $t_{1}$ in Figure 4 may not contains the region cover by $s_{1}, s_{2}, s_{3}$. In order to respect the sphere-tree properties, we could replace their parent spheres by the minimal bounding spheres of each set. However, the minimal covering sphere computation is not very efficient since the problem is related to the minimal enclosing ball of a set of points in $d$ dimension [10]. In order to maintain the reversibility of multiresolution representations of the original object, we proposed an original approach, which consist in extending the radius of parent spheres. 

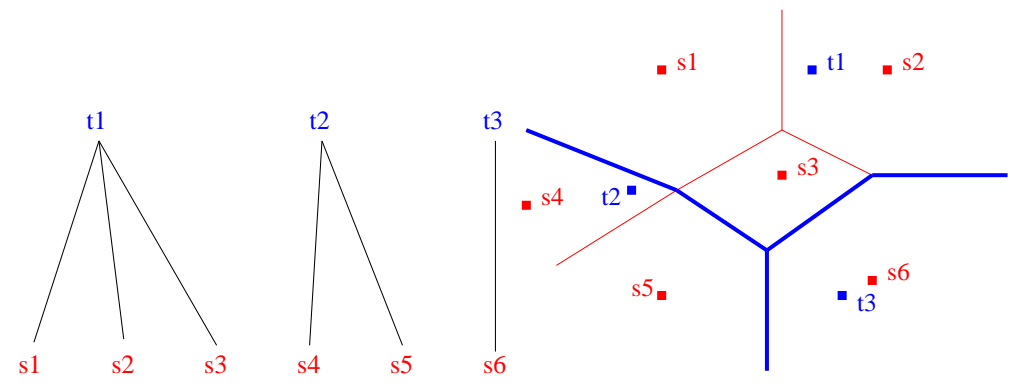

Fig. 5. Sphere-tree reduction of the previous DAG. We can also create the recovering diagram, extending each cell in $\mathcal{C}_{N-1}$ (associated with a sphere $t_{j}$ ) as the union of children cells.

Theorem 2. Let a sphere $t$ and his set of children spheres $\left\{s_{i}\right\}$. The sphere $t$ is a minimal bounding sphere centered at $c_{t}$ for its children spheres if its radius $r_{t}$ is extended by $r_{t}^{\prime}=\sigma_{t}^{\prime}(\max )$, with $\max$ is the child sphere where $\sigma_{t}^{\prime}$ is maximal.

Proof. For two spheres $s, t$, by Definition 3 we know that $t$ entirely covers $s$ if $\sigma_{t}^{\prime}(s) \leq 0$. Moreover, if $\sigma_{t}^{\prime}(s)=0$ we have $d\left(\mathcal{S}\left(c_{s}\right), c_{t}\right)+r_{s}=r_{t}$, so the sphere $t$ is the minimal bounding sphere for $s$ centered at $c_{t}$. Let $r_{t}^{\prime}$ the quantity to add to $r_{t}$ in order to have a covering of $s$ by $t$. So, we have:

$$
d\left(\mathcal{S}\left(c_{s}\right), c_{t}\right)-\left(r_{t}+r_{t}^{\prime}\right)+r_{s}=0 \Leftrightarrow r_{t}^{\prime}=d\left(\mathcal{S}\left(c_{s}\right)\right), c_{t}-r_{t}+r_{s} \Leftrightarrow r_{t}^{\prime}=\sigma_{t}^{\prime}(s)
$$

So $\sigma_{t}^{\prime}(s)$ defines the extension quantity. Now, for each parent sphere $t_{j}$, we search among its children $s_{i}$ the sphere $s_{\max }$, where $\sigma_{t}^{\prime}\left(s_{\max }\right)$ is maximal. Extending the radius with this value, we substract $\sigma_{t}^{\prime}\left(s_{\max }\right)$ at all $\sigma_{t}^{\prime}(i)$, so we have $\sigma_{t}^{\prime}(i) \leq 0$ for all children spheres $s_{i}$ and $\sigma_{t}^{\prime}(\max )=0$. Hence $t$ becomes the minimal bounding sphere of its children centered at $c_{t}$

The Algorithm 2 adds this extending process to the reversible sphere-tree computation. As radii have been extended, we have to modify $\mathcal{F}_{N-1}$ by a reverse reconstruction of the object with the new set of spheres. For $n$ spheres in $\mathcal{A M}_{N}$, the linking computation and the extension of the spheres are in $O(n)$, and the reconstruction process in $O\left(c^{d}\right)$. As the number of spheres $n$ is lower than the size of the object $\left(c^{d}\right)$, the time for one iteration stays in $O\left(c^{d}\right)$. So this process is also in $O\left(c^{d}\right)$ like the Sphere-DAG Computation (cf Theorem 1).

We now have a sphere-tree which respects the covering condition. Nevertheless, it needs a modification of spheres radii, although the spheres were first defined to ensure the distribution of error along the object. So the extension of radii disturbs the reversibility of the model we first create. On the other hand, when the value $\sigma_{t}^{\prime}\left(s_{\max }\right)$ we add to radius are negative, the parents spheres are going to be reduced, generating an improvement of representation tightness. Moreover, as we simplify the sphere-tree by deleting the spheres without child, 


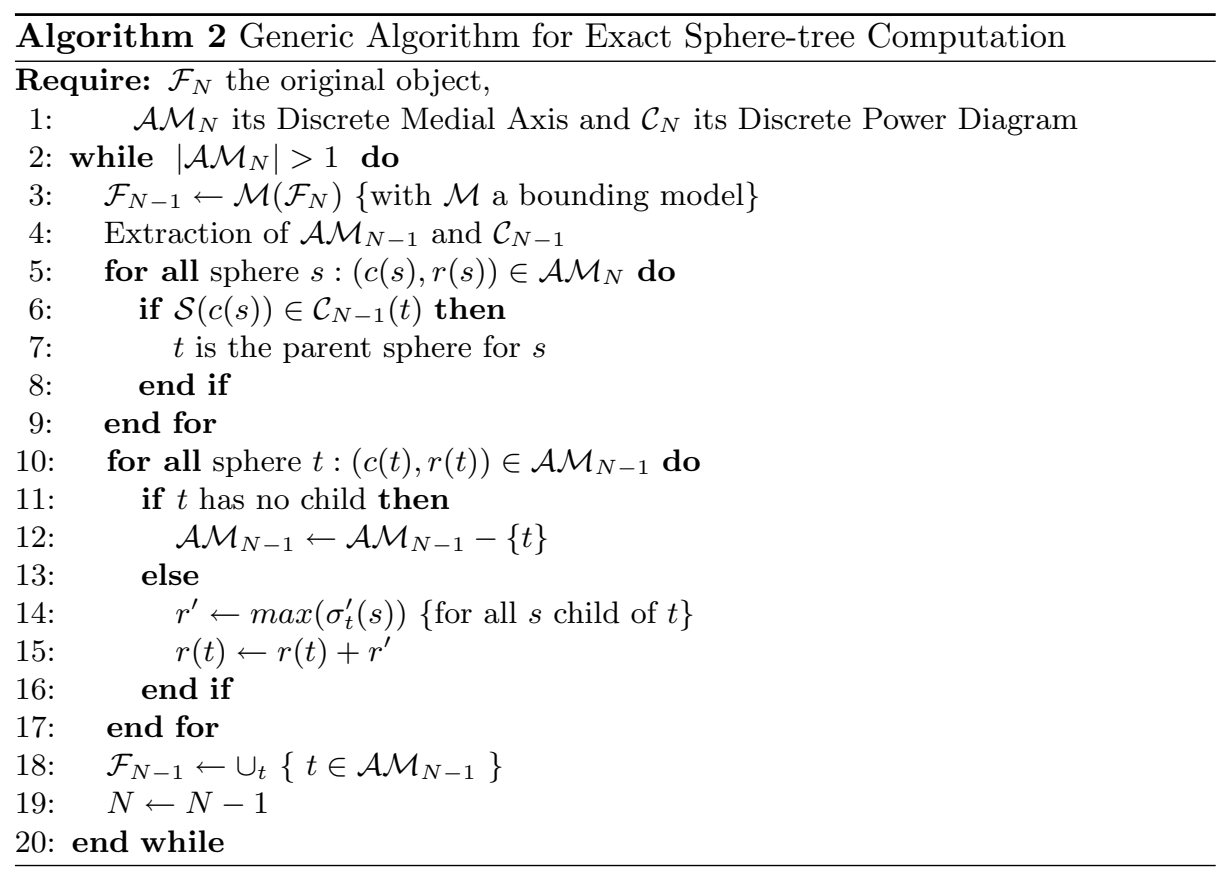

this algorithm may reduce the depth of the tree. The following section demonstrates these observations on real images. In order to reduce the error with the original object, we can also evaluate the radii extension for each node with its set of leaves (i.e. spheres of the original object.

\section{Experiments}

This section presents a comparison between the Reversible and the Extended Algorithm, with experiments on many 3D discrete objects in .vol or .longvol format, defined in the simplevol library. Data Transformation, Reduce Discrete Medial Axis Extraction and Discrete Power Diagram are computed with the usage of the MAEVA Toolkit ${ }^{1}$.

Figure 6 shows differences between both algorithms on objects Al.100.vol and ArachnidWarrior.100. vol ${ }^{2}$. The left graph presents the number of spheres for each level, in the right one we have a percentage of error between the representation for one level respect and the original level. This error is computed by the Hamming Distance that we can efficiently compute in our discrete model. As the experimental objects have different number of voxels, we prefer represent the Hamming distance by a percentage representing the error volume added to the original object.

\footnotetext{
${ }^{1}$ Simplevol and MAEVA Toolkit are available on http://gforge.liris.cnrs.fr/

2 These objects are available on http://www.tc18.org/
} 

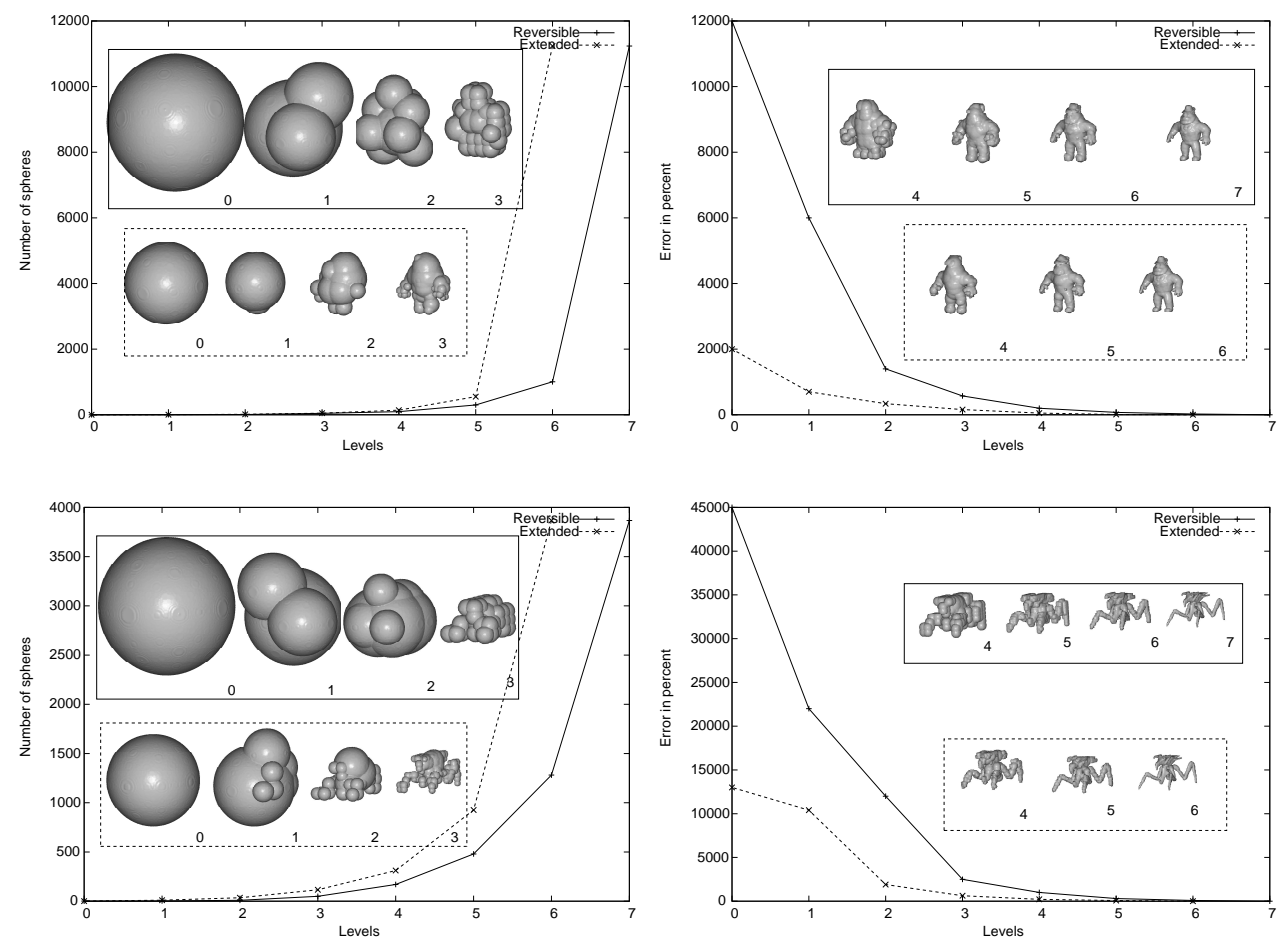

Fig. 6. Experiments for Al.100.vol and ArachnidWarrior.100.vol.

\subsection{Reverse Algorithm}

The description of our discrete model in the pyramid (cf Section 2.1) shows the fact that voxels for superior levels are $f^{d}$ times bigger than voxels at the lower level, so their number becomes smaller, and the Medial Axis of the associated object contains less spheres, as we can see in Figure 6. However, the distance computation is computed between two voxel centers in the discrete approach (here we use the square-euclidean distance), but this distance also depends of the voxels size and it is not adapted of the original object. That's why we scale the spheres to the finer resolution level, by a reconstruction process.

Moreover, at upper levels, the variability of radii is lower, as the interval of radii becomes smaller. This range remains low at reconstruction, because all the radius of spheres are increased with the same rate (at one level). So the spheretree looks like an octree, as many spheres have the same radius. Nevertheless, the reconstruction ratio at an upper level is very high, and the spheres overestimate the object geometry on this level. 


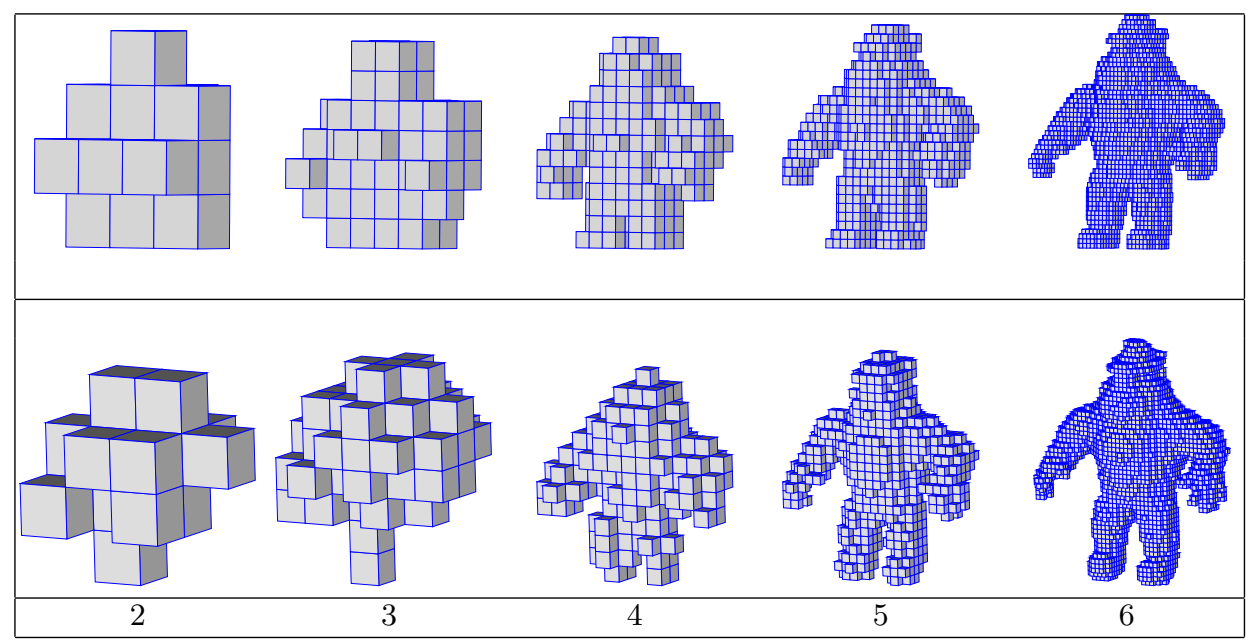

Fig. 7. Comparison between the reversible pyramid and the dynamic reconstructions of the extended version.

\subsection{Extended Sphere-tree}

Experiments for the extended sphere-tree construction show that the dynamic reconstruction before the extension of radius simplifies upper levels. As we delete spheres without child, the reduction of sphere number is faster than in the reversible algorithm. Moreover, in Algorithm 2 we have proposed a reconstruction of the upper level just after the extension of the radius of its spheres, and experiments show that this process reduces the increasing of error which is higher in the reversible case (cf Figure 7). In order to reduce the incidence of the final reconstruction process, we propose to fit one more time the spheres by $\sigma_{t}^{\prime}\left(s_{\max }\right)$ after the increasing. As the reconstruction has produced spheres which overestimate the object, this final extension reduces the error.

\section{Conclusion and Future Works}

In this paper, we have presented an original method for a sphere-tree construction in discrete geometry. Its construction is based on the Discrete Medial Axis of the object, as best algorithms in computational geometry $[13,6,7]$, but we benefit of the fact that we can efficiently extract a reversible skeleton. Hence we have built a pyramid of object and we extract a reversible set of spheres at each level. Then the linking of spheres at different levels is solved using properties of power diagrams at each level, as we established a relation between the interaction and the covering of spheres. In order to have a sphere-tree ensuring the covering conditions, we propose a fast method to obtain bounding spheres of nodes, with an extension of radii, instead of using complex optimization heuristics [7].

Moreover in discrete geometry, we can exactly measure the error with a Hamming distance instead of a Haussdorf estimation. Experiments show that 
the reversible algorithm produces reversible representations at each level, nevertheless the increasing of error respect to the original object is bigger when we return at the original resolution. However, the extended algorithm solves this problem, and the error increasing is reduced, as we build a dynamic hierarchy.

The methods we have presented here are generic, they can be used in dimension $d$, and for any including model of the pyramid. In future works, we may extend these methods for generic models. We can also imagine other heuristics in order to get a good sphere-tree, like in section 3.3, replacing the extending treatment. However, the representation of the object is clearly modified and the final result not depend on it in this case. Future works can be oriented to the construction of other including models (like morphological models) in order to reduce the consequences of the extension process for a better topology maintenance.

\section{References}

1. N. Amenta, S. Choi, and R. Krishna Kolluri. The power crust, unions of balls, and the medial axis transform, July 222000.

2. D. Attali and H. Edelsbrunner. Inclusion-exclusion formulas from independent complexes. 2007.

3. F. Aurenhammer. Power diagrams: Properties, algorithms, and applications. SIAM Journal on Computing, 16(1):78-96, February 1987.

4. H. Blum. A transformation for extracting new descriptors of shape. In W. WhatenDunn, editor, Models for the Perception of Speech and Visual Form, pages 362-380. MIT Press, Cambridge, MA, 1967.

5. Boissonnat, Cerezo, Devillers, Duquesne, and Yvinec. An algorithm for constructing the convex hull of a set of spheres in dimension d. CGTA: Computational Geometry: Theory and Applications, 6, 1996.

6. G. Bradshaw and C. O'Sullivan. Sphere-tree construction using dynamic medialaxis approximation. In Stephen N. Spencer, editor, Proceedings of the 2002 ACM SIGGRAPH Symposium on Computer Animation (SCA-02), pages 33-40, New York, July 21-22 2002. ACM Press.

7. G. Bradshaw and C. O'Sullivan. Adaptive medial-axis approximation for spheretree construction. ACM Transactions on Graphics, 23(1):1-26, January 2004.

8. D. Coeurjolly and A. Montanvert. Optimal separable algorithms to compute the reverse euclidean distance transformation and discrete medial axis in arbitrary dimension. IEEE transactions on pattern analysis and machine intelligence, VOL. 29, NO. 3, MARCH 2007, aug 222007.

9. C. Dingliana. Real-time collision detection and response using sphere-trees. Technical report, March 021999.

10. Jacob E. Goodman and Joseph O'Rourke, editors. Handbook of Discrete and Computational Geometry. CRC Press, 1997.

11. S. Gottschalk, M. C. Lin, and D. Manocha. OBBTree: A hierarchical structure for rapid interference detection. Computer Graphics, 30(Annual Conference Series):171-180, 1996.

12. T. He and A. Kaufman. Collision detection for volumetric objects. In IEEE Visualization '97, October 1997. 
13. P. Hubbard. Approximating polyhedra with spheres for time-critical collision detection. ACM Transactions on Graphics, 15(3):179-210, July 1996.

14. I. J. Palmer and R. L. Grimsdale. Collision detection for animation using spheretrees. Computer Graphics Forum, 14(2):105-116, June 1995.

15. J. Pfaltz and A. Rosenfeld. Computer representation of planar regions by their skeletons. Communications of the ACM, 10(2):119-122, February 1967.

16. S. Prevost, L. Lucas, and E. Bittar. Multiresolution and shape optimization of implicit skeletal model. In V. Skala, editor, WSCG 2001 Conference Proceedings, 2001.

17. S. Quinlan. Efficient distance computation between non-convex objects. In Edna Straub and Regina Spencer Sipple, editors, Proceedings of the International Conference on Robotics and Automation. Volume 4, pages 3324-3330, Los Alamitos, CA, USA, May 1994. IEEE Computer Society Press.

18. G. van den Bergen. Efficient collision detection of complex deformable models using AABB trees. Journal of Graphics Tools: JGT, 2(4):1-14, 1997. 